\title{
Hipotermia e banho do recém-nascido nas primeiras horas de vida
}

\author{
Hypothermia and the newborn's bath in the first hours of life \\ Hipotermia y el baño del recién nascido en las primeras horas de vida
}

\section{Como citar este artigo:}

Ruschel LM, Pedrini DB, Cunha MLC. Hipotermia e banho do recém-nascido nas primeiras horas de vida. Rev Gaúcha Enferm. 2018;39:e20170263. doi: https://doi.org/10.1590/19831447.2018.20170263.

\footnotetext{
a Universidade Federal do Rio Grande do Sul (UFRGS). Escola de Enfermagem. Porto Alegre, Rio Grande do Sul, Brasil.

- Universidade Federal do Rio Grande do Sul (UFRGS). Programa de Pós-graduação em Enfermagem. Porto Alegre, Rio Grande do Sul, Brasil.
}

\section{RESUMO}

Objetivo: analisar a ocorrência de hipotermia em recém-nascidos antes e após o banho nas primeiras horas de vida. Método: estudo transversal, no qual se verificou a temperatura axilar de recém-nascidos antes do banho, após o banho, 30 minutos após o banho e 60 minutos, no Centro Obstétrico. Na análise estatística utilizou-se os Testes Qui-Quadrado, t de Student e Mann-Whitney, com a =0,05.

Resultados: Foram incluídos 149 recém-nascidos no estudo, evidenciando-se a prevalência de hipotermia neonatal em 40,3\% dos casos, tendo associação com significância estatística $(p<0,001)$ entre a ocorrência de hipotermia neonatal em todos os momentos de verificação de temperatura axilar. Constatou-se correlação estatística significativa entre as variáveis: temperatura da sala de parto e a verificação da temperatura 60 minutos após o banho $(p=0,032)$.

Conclusões: Conclui-se que o primeiro banho pode ser adiado para favorecer a adaptação do neonato ao ambiente extrauterino, prevenindo a ocorrência de hipotermia neonatal.

Palavras-chave: Recém-nascido. Banhos. Hipotermia.

\section{ABSTRACT}

Objective: to analyze the occurrence of hypothermia in neonates before and after bathing in the first hours of life.

Method: a cross-sectional study in which the axillary temperature of newborns before bathing, after bathing, 30 and 60 minutes after bathing was verified at an Obstetric Center. In the statistical analysis, the Chi-Square, Student's t and Mann-Whitney tests were used, with $a=0.05$

Results: A total of 149 newborns were included in the study, showing the prevalence of neonatal hypothermia in $40.3 \%$ of the cases, with a statistically significant association $(p<0.001)$ between the occurrence of neonatal hypothermia at all axillary temperature assessments. A statistically significant correlation was found between the variables: room temperature and temperature verification 60 minutes after bath $(p=0.032)$

Conclusions: It is concluded that the first bath can be postponed to favor the adaptation of the neonate to the extrauterine environment, preventing the occurrence of neonatal hypothermia.

Keywords: Infant, newborn. Baths. Hypothermia.

\section{RESUMEN}

Objetivo: analizar la ocurrencia de hipotermia en recién nacidos antes y después del baño en las primeras horas de vida.

Método: estudio transversal, en el cual se verificó la temperatura axilar de recién nacidos antes del baño, después del baño, 30 minutos después del baño y 60 minutos, en el Centro Obstétrico. En el análisis estadístico se utilizaron las pruebas Qui-cuadrado, t de Student y Mann-Whitney, con a =0,05.

Resultados: Se incluyeron 149 recién nacidos en el estudio, evidenciándose la prevalencia de hipotermia neonatal en el 40,3\% de los casos, teniendo asociación con significancia estadística $(p<0,001)$ entre la ocurrencia de hipotermia neonatal en todos los momentos de verificación de temperatura axilar. Se constató correlación estadística significativa entre las variables: temperatura de la sala de parto y la verificación de la temperatura 60 minutos después del baño $(p=0,032)$.

Conclusiones: Se concluye que el primer baño puede ser pospuesto para favorecer la adaptación del neonato al ambiente extrauterino, previniendo la ocurrencia de hipotermia neonatal.

Palabras clave: Recién nacido. Baños. Hipotermia. 


\section{— INTRODUÇÃO}

O banho do recém-nascido (RN) é um procedimento rotineiro que segue tradição cultural, tendo como objetivo remover resíduos da pele e reduzir a colonização da mesma. A recomendação desse procedimento necessita ser baseada em evidências para evitar o prejuízo da pele do RN, devido essa se modificar rapidamente em função do processo adaptativo do meio intrauterino para o meio extrauterino ${ }^{(1)}$.

O primeiro banho do recém-nascido é um cuidado que gera muitas controvérsias com relação à sua realização. Um estudo sugere que o primeiro banho deve ser adiado por no mínimo 24 horas, juntamente com a não remoção precoce da vernix caseosa, que traria uma série de benefícios ao RN, dentre eles: adequada termorregulação, hidratação da pele, ausência de descamação da pele, redução de eritema tóxico neonatal, cicatrização de feridas e colonização da pele por bactérias não patogênicas (2).

Ao considerar tais benefícios, preconiza-se que a separação da mãe e do RN nas primeiras horas de vida seja evitada, favorecendo, assim, o contato pele a pele. Tal prática auxilia em vários mecanismos de autorregulação do recém-nascido, dentre eles a manutenção da temperatura corporal constante. Nesse sentido, a realização de procedimentos de cuidado precoces pode interromper o processo adaptativo ao ambiente extrauterino ${ }^{(3)}$.

De acordo com as diretrizes para a organização da atenção integral ao recém-nascido no Sistema único de Saúde, deve-se assegurar o contato pele a pele imediato e contínuo, estimular o aleitamento materno na primeira hora de vida e postergar procedimentos de rotina (como o banho, por exemplo) para os RN a termo com ritmo respiratório normal, tônus normal e sem líquido meconial ${ }^{(4)}$. A permanência e contato do binômio mãe/bebê propicia diversos benefícios a ambos, tais como a prevenção da hipotermia neonatal e o estímulo ao aleitamento materno precoce ${ }^{(5-6)}$.

A justificativa do estudo se deve ao fato de haver controvérsias na literatura quanto ao momento ideal do primeiro banho do recém-nascido, tendo em vista o risco para hipotermia presente no período de transição do nascimento para vida neonatal. Considerando que em nosso meio o banho é realizado nas primeiras duas horas de vida do RN e que existem recomendações de que esse procedimento seja adiado para um período de 6 h após o nascimento ${ }^{(7)}$, ou até mesmo recomendando-se que seja adiado por $24 \mathrm{~h}^{(2)}$ com o propósito de evitar hipotermia. Acredita-se que uma investigação criteriosa metodologicamente seja necessária para preencher essa lacuna do conhecimento científico, o que se propõe no atual estudo.
Nesse sentido, ao considerar as informações sobre a realização do banho nas primeiras horas de vida, propõe-se o seguinte questionamento: "Qual o efeito do primeiro banho sobre a temperatura axilar do recém-nascido nas primeiras horas de vida?". Portanto, este estudo tem por objetivo analisar a ocorrência de hipotermia em recém-nascidos antes e após o banho nas primeiras horas de vida.

\section{MÉTODOS}

Trata-se de um estudo transversal. A presente pesquisa pertence a um projeto de dissertação de mestrado vinculado ao Programa de Pós-graduação em Enfermagem da Universidade Federal do Rio Grande do Sul (UFRGS) ${ }^{(8)}$. O presente estudo foi realizado nas salas de parto e sala de admissão do recém-nascido da Unidade Centro Obstétricas (UCO) em um hospital universitário situado no município de Porto Alegre, Rio Grande do Sul, Brasil. A amostra foi constituída por recém-nascidos cujo parto foi realizado no referido hospital. Foram incluídos no estudo os RN em bom estado geral, com idade gestacional $\geq 35$ semanas e peso $\geq 2000$ g. Foram excluídos os recém-nascidos que necessitassem internar na UTI Neonatal, os recém-nascidos filhos de mães com soropositividade para HIV e filhos de mães com qualquer infecção do grupo STORCH. Para o cálculo amostral, considerou-se uma proporção de 10\%, poder de 80\% e índice de significância de 5\%. Dessa forma, o número de participantes para compor o presente estudo foi de 149 recém-nascidos. O cálculo foi realizado com auxílio do software WINPEPI.

A coleta de dados ocorreu nas salas de parto e na sala de admissão do RN da Unidade Centro Obstétrico (UCO) de um hospital universitário, nos meses de abril a outubro de 2017, sendo estratificada em quatro momentos. Inicialmente, foi realizada a verificação da temperatura axilar com termômetro clínico "Medlevenson ${ }^{\circledR "}$ por um minuto antes do banho do recém-nascido (momento 1). Tal período ocorreu na sala de admissão do RN, com o mesmo sob o berço de calor radiante. Após, foi verificada a temperatura axilar, imediatamente após o banho (momento 2), assim que o corpo do RN estivesse seco depois do procedimento. A temperatura axilar também foi verificada após transcorridos 30 minutos (momento 3) e 60 minutos do término do banho (momento 4). Salienta-se que os momentos 1, 2 e 3 de verificação da temperatura axilar ocorreram na Sala de Admissão do RN, enquanto que o momento de verificação 4 ocorreu na Sala de Recuperação, onde o recém-nascido retorna para a companhia de sua mãe.

Os dados coletados, assim como dados clínicos e sociodemográficos da mãe e do $\mathrm{RN}$, foram retirados dos re- 
gistros de enfermagem contidos em prontuário eletrônico. A presente pesquisa utilizou a definição de hipotermia da Associação Americana de Pediatras ${ }^{(9)}$, na qual a temperatura axilar $\leq 36,4^{\circ} \mathrm{C}$ corresponde à hipotermia neonatal e a faixa térmica entre $36,5^{\circ}$ a $37,5^{\circ} \mathrm{C}$ corresponde a normotermia. Foram seguidas as normas e orientações da Resolução 466 de $2012^{(10)}$ sobre pesquisa com seres humanos. O presente estudo foi aprovado pelo Comitê de Ética da Instituição sob o parecer número 160026. Todos os pais responsáveis pelos RN assinaram o Termo de Consentimento Livre e Esclarecido.

De acordo com a rotina do hospital onde foi realizado o estudo, o RN nasce nas salas de parto e/ou cesárea na Unidade Centro Obstétrico. Em situações normais, o mesmo (após avaliação do pediatra e após a pesagem) é colocado sobre o tórax/abdome da mãe e permanece em contato pele a pele por pelo menos uma hora, sendo estimulado o aleitamento na primeira hora de vida com o auxílio da equipe assistente.

Ao término do período de contato pele a pele, a mãe é levada para a sala de recuperação e o recém-nascido é levado à Sala de Admissão para receber os primeiros cuidados rotineiros, tais como, exame físico e o banho.

Para este estudo considerou-se o primeiro momento de verificação da temperatura axilar antes do banho, com o RN despido e sob o berço de calor radiante, quando se verificou a temperatura axilar por um minuto com termômetro clínico "Medlevenson ${ }^{\oplus " . ~ A ~ r o t i n a ~ i n s t i t u c i o n a l ~ p a r a ~}$ o primeiro banho preconiza que a temperatura axilar do neonato deve ser de $36,8^{\circ} \mathrm{C}$ para a realização do procedimento, estando a temperatura da água do banho em $38^{\circ} \mathrm{C}$ e a temperatura do ambiente entre $25^{\circ} \mathrm{C}$ e $27^{\circ} \mathrm{C}$. A temperatura da água do banho e do ambiente foi verificada por termômetro digital da marca "Incoterm" ${ }^{\oplus "}$.

O segundo momento de verificação da temperatura axilar foi imediatamente após o banho (logo após a secagem e estando o RN sob o berço de calor radiante). $\mathrm{O}$ terceiro momento de verificação foi depois de transcorridos 30 minutos do término do banho, quando o RN ainda estava na Sala de Admissão (já vestido) e sob uma fonte externa de calor. O quarto momento de verificação da temperatura foi depois de decorridos 60 minutos do término do banho, quando o recém-nascido se encontrava na sala de recuperação (acompanhado por sua mãe), estando em contato pele-a-pele com a mesma ou em berço comum (vestido) ao lado do leito materno.

Para a análise dos dados utilizou-se o software estatístico SPSS versão 18.0. Inicialmente os dados foram tratados descritivamente por meio de frequências, média, desvio padrão, mediana e intervalo interquartílico. Para a análise estatística, foi utilizado o Teste Qui-Quadrado para verificar associação entre as categorias de hipotermia e fatores estudados, tais como as variáveis de intervalos de tempo de duração do primeiro banho e do período de realização do primeiro banho. O Teste t de Student e o Teste de Mann-Whitney, de acordo com a distribuição das variáveis quantitativas, foram usados para comparar as médias, ou distribuições, das covariáveis estudadas, tais como: a idade gestacional, o peso de nascimento e os momentos de verificação de temperatura axilar. O nível de significância estatístico aceito foi de $a=0,05$. Utilizou-se a correlação de Spearman, na qual foi verificado o grau de relação das variáveis quantitativas, como: a temperatura da sala de parto, temperatura da sala de admissão e a temperatura da água com os momentos de verificação de temperatura axilar.

\section{口 RESULTADOS}

Foram incluídos no estudo 149 recém-nascidos. Não houve perdas e/ou negativas de consentimento. Com relação aos dados demográficos da população materna, constatou-se que $63(42,3 \%)$ das mães estavam na faixa etária de 18 a 24 anos, 56 (37,6\%) possuíam ensino médio completo, 98 (65,8\%) eram primíparas e a maior frequência de partos foram vaginais - $93(62,4 \%)$.

Evidenciou-se a ocorrência de hipotermia em 60 neonatos $(40,3 \%)$ em algum dos quatro momentos de verificação de temperatura axilar. Desses momentos, observou-se prevalência de hipotermia em 12\% dos casos antes do banho, 11\% imediatamente após o banho, $6 \%$ em 30 minutos após o término do banho e 11,4\% no período de 60 minutos após o banho.

Para melhorar a análise, os dados foram estratificados em dois grupos, sendo um grupo de hipotermia e o outro de normotermia. Buscou-se investigar associação entre peso, idade gestacional e os momentos de verificação da temperatura, porém não foi observada significância estatística (Tabela 1).

Com relação às variáveis independentes citadas, buscou-se investigar a associação entre os quatro momentos de verificação de temperatura axilar com a ocorrência ou não de hipotermia neonatal, tendo sido evidenciada significância estatística em todos os momentos de verificação (Tabela 2).

Ao analisar as temperaturas das salas de parto, foi observada uma mediana de $23,9^{\circ} \mathrm{C}\left(22,5-24,8^{\circ} \mathrm{C}\right)$, ou seja, cerca de 80\% dos recém-nascidos foram expostos a uma temperatura ambiental inferior a $25^{\circ} \mathrm{C}$. Com relação à sala de admissão do RN, foi visualizada uma mediana de $25,8^{\circ} \mathrm{C}\left(24,9-26,4^{\circ} \mathrm{C}\right)$. Observa-se, assim, que a sala de admissão obteve temperaturas mais elevadas em relação às salas de parto, porém cerca de $20 \%$ dos RN foram expostos à temperatura ambiental inferior a $25^{\circ} \mathrm{C}$ durante os primeiros cuidados de admissão. 
Tabela 1 - Dados dos recém-nascidos relativos a variáveis da pesquisa (n=149). Porto Alegre, RS, 2017

\begin{tabular}{|c|c|c|c|}
\hline Características & Normotermia $\mathbf{n = 8 9}$ & Hipotermia $n=60$ & $\mathbf{p}^{*}$ \\
\hline Idade Gestacional (semanas) & $\begin{array}{c}39+2(38+5-40+2) \\
{[36-41]}\end{array}$ & $\begin{array}{c}40(38+2-41) \\
{[35-42]}\end{array}$ & 0,616 \\
\hline Peso (g) & $\begin{array}{c}3365(3052-3647) ; \\
{[2515-4595]}\end{array}$ & $\begin{array}{c}3332(3047-3601) \\
{[2115-4065]}\end{array}$ & 0,500 \\
\hline Momento $1\left({ }^{\circ} \mathrm{C}\right)$ & $\begin{array}{c}37(36,8-37,1) \\
{[36,5-38,1]}\end{array}$ & $\begin{array}{c}36,5(36,2-36,9) \\
{[35,2-37,8]}\end{array}$ & 0,200 \\
\hline Momento $2\left({ }^{\circ} \mathrm{C}\right)$ & $\begin{array}{c}36,9(36,7-37,1) \\
{[36,5-37,8]}\end{array}$ & $\begin{array}{c}36,6(36,4-37) \\
{[35,6-37,2]}\end{array}$ & 0,200 \\
\hline Momento $3\left({ }^{\circ} \mathrm{C}\right)$ & $\begin{array}{c}37(36,8-37,2) \\
{[37-38]}\end{array}$ & $\begin{array}{c}36,7(36,5-36,9) ; \\
{[36-38]}\end{array}$ & 0,200 \\
\hline Momento $4\left({ }^{\circ} \mathrm{C}\right)$ & $\begin{array}{c}37(36,8-37,1) \\
\quad[36,5-38,2]\end{array}$ & $\begin{array}{c}36,6(36,4-36,9) ; \\
{[35,7-38,4]}\end{array}$ & 0,200 \\
\hline
\end{tabular}

Fonte: Dados da pesquisa, 2017.

Mediana (amplitude interquartil: P25 e P75) e [mínimo e máximo].

*Teste t de Student.

Tabela 2 - Prevalência de Hipotermia nos momentos de verificação de temperatura axilar do recém-nascido. Porto Alegre, RS, 2017

\begin{tabular}{|c|c|c|c|}
\hline & Hipotermia $n=60$ & Normotermia $n=\mathbf{8 9}$ & $p^{*}$ \\
\hline Momento 1 & $\begin{array}{c}36,7(36,2-36,9) \\
{[35,2-37,8]}\end{array}$ & $\begin{array}{c}37(36,8-37,1) \\
{[36,5-38,1]}\end{array}$ & $<0,001$ \\
\hline Momento 2 & $\begin{array}{c}36,7(36,4-37) \\
{[35,6-37,6]}\end{array}$ & $\begin{array}{c}36,9(36,7-37,1) \\
{[36,5-37,8]}\end{array}$ & 0,001 \\
\hline Momento 3 & $\begin{array}{c}36,8(36,5-37) \\
{[36-38]}\end{array}$ & $\begin{array}{c}37(36,8-37,2) ; \\
{[37-38]}\end{array}$ & $<0,001$ \\
\hline Momento 4 & $\begin{array}{c}36,6(36,4-36,9) \\
{[35,7-38,4]}\end{array}$ & $\begin{array}{c}37(36,8-37,1) \\
{[36,5-38,2]}\end{array}$ & $<0,001$ \\
\hline
\end{tabular}

Fonte: Dados da Pesquisa, 2017.

Mediana (amplitude interquartil: P25 e P75) e [mínimo e máximo].

*Teste de Mann-Whitney.

Ao considerar as características do primeiro banho do recém-nascido, foi avaliada a temperatura da água do banho, o período em que foi realizado o primeiro banho e o tempo de duração do mesmo. Considerando a temperatura da água do banho, a maioria das verificações esteve dentro e/ou próxima à temperatura preconizada pela rotina assistencial do banho da instituição onde foi realizada a presente pesquisa, ou seja, $38^{\circ} \mathrm{C}$.

O momento de realização do primeiro banho foi estratificado em: entre 1 a $2 \mathrm{~h}$ de vida, entre 2 a $3 \mathrm{~h}$ de vida e entre 3 a 4 h de vida. Constatou-se que a maioria dos RN recebeu o primeiro banho entre 1 a 2 horas de vida. Esses recém-nascidos, que receberam o primeiro banho nesse intervalo de tempo, representam 91,7\% dos 60 casos de hipotermia neonatal decorrentes do banho observados nesse estudo. Buscou-se investigar associações entre o período de realização do primeiro banho e a ocorrência ou não de hipotermia, porém não foram encontradas associações estatisticamente significativas.

O tempo de duração do banho foi estratificado em: 1 a 2 min, 2 a 3 min, 3 a 4 min, 4 a 5 min e 5 min ou mais. Foi observado que a maioria dos banhos teve duração de 2 a 3 minutos. Buscou-se avaliar possíveis associações entre o tempo de duração do banho e a ocorrência de hipotermia ou não, porém não foram encontradas associações com significância estatística (Tabela 3). 
Tabela 3 - Período de realização do primeiro banho do recém-nascido ( $n=149$ ). Porto Alegre, RS, 2017

\begin{tabular}{cccccccc}
\hline Intervalo & Hipotermia & $\%$ & Normotermia & $\%$ & Total & $\%$ & $\boldsymbol{p}^{*}$ \\
$1 \rightarrow 2 \min$ & 10 & 45,5 & 12 & 54,5 & 22 & 100 & \\
$2 \rightarrow 3 \min$ & 15 & 36,6 & 26 & 63,4 & 41 & 100 & \\
$3 \rightarrow 4 \min$ & 13 & 37,1 & 22 & 62,9 & 35 & 100 & 0,472 \\
$4 \rightarrow 5 \min$ & 11 & 34,4 & 21 & 65,6 & 32 & 100 & \\
5 min ou mais & 11 & 57,9 & 8 & 42,1 & 19 & 100 & \\
\hline
\end{tabular}

Fonte: Dados da Pesquisa, 2017.

*Teste Qui-Quadrado (Teste de Pearson)

Foram analisadas possíveis associações entre as variáveis: temperatura da sala de parto, temperatura da sala de admissão, temperatura da água e momentos de verificação de temperatura axilar. Encontrou-se correlação significativa, porém fraca, entre as variáveis: temperatura da sala de parto e momento quatro de verificação de temperatura axilar, ou seja, após transcorridos 60 minutos do término do primeiro banho, quando o RN já estava novamente na companhia de sua mãe na Sala de Recuperação. Tais dados podem ser visualizados na Tabela 4.

Tabela 4 - Correlação entre variáveis do recém-nascido ( $n=149)$. Porto Alegre, RS, 2017

\begin{tabular}{ccccccccc}
\hline Variáveis & \multicolumn{2}{c}{ Momento $\mathbf{1}$} & \multicolumn{2}{c}{ Momento $\mathbf{2}$} & \multicolumn{2}{c}{ Momento 3 } & \multicolumn{2}{c}{ Momento 4} \\
Sala de Parto & $r$ & $P$ & $R$ & $p$ & $r$ & $P$ & $r$ & $p$ \\
Sala Admissão & $-0,100$ & $(0,223)$ & $-0,088$ & $(0,288)$ & 0,021 & $(0,797)$ & $\mathbf{0 , 1 7 5}$ & $\mathbf{( 0 , 0 3 2 )}$ \\
Temperatura água & $-0,067$ & $(0,414)$ & 0,175 & $(0,033)$ & 0,118 & $(0,151)$ & 0,114 & $(0,167)$ \\
\hline
\end{tabular}

Fonte: Dados da Pesquisa, 2017.

$r$ (p): Correlação de Spearman.

\section{DISCUSSÃO}

Por meio do presente estudo foi possível analisar a ocorrência de hipotermia em recém-nascidos antes e após o banho nas primeiras horas de vida. Verificou-se a prevalência de hipotermia neonatal em 40,3\% da amostra de RN estudados. O banho do RN é um tema controverso. Discussões sobre como realizar o procedimento e qual o momento mais apropriado nos primeiros dias de vida ainda são presentes na rotina de cuidados neonatais ${ }^{(11)}$. Com o intuito de prevenir a hipotermia neonatal, é recomendado que o primeiro banho seja realizado após seis horas de vida do recém-nascido(7).

Em estudo realizado em quatro regiões da Etiópia, por meio de entrevistas executadas em visitas domiciliares por profissionais da saúde, demonstrou-se que o adiamento do primeiro banho contribuiu para a manutenção da temperatura corporal do neonato, além de manter os recém- -nascidos vivos devido ao maior período de contato com a mãe ${ }^{(12)}$. Corroborando a esses achados, estudo realizado nos Estados Unidos, demonstrou que o adiamento do primeiro banho do neonato em até 12 horas contribui para reduzir os riscos de hipotermia neonatal decorrentes da separação do binômio mãe/bebê, trazendo diversos benefícios para ambos ${ }^{(5)}$.

Na presente pesquisa, observou-se que as temperaturas das salas de parto estavam abaixo da faixa térmica preconizada pela literatura, sendo que grande número dos RN em estudo foi exposto a temperaturas inferiores a $25^{\circ} \mathrm{C}$ ao nascimento e enquanto permaneciam em contato pele-a-pele com suas mães. Na sala de admissão, por sua vez, as temperaturas observadas foram mais elevadas e estavam de acordo com as recomendações de cuidado termal do recém-nascido ${ }^{(13)}$.

As temperaturas de salas de parto mais baixas elevam os riscos de perda de calor por condução e convecção no 
RN, assim, estudo demonstrou que quando recém-nascidos são expostos a temperaturas mais baixas (de aproximadamente $23^{\circ} \mathrm{C}$ ), os mesmos tendem a apresentar hipotermia de intensidade moderada a severa nos cuidados de admissão. Desse modo, é recomendado o aumento da temperatura da sala no momento do procedimento com o intuito de prevenir a hipotermia materna e neonatal(14).

Com relação às características do banho do recém-nascido, foi observado que a temperatura da água, utilizada para o primeiro banho de imersão, manteve-se dentro da faixa térmica preconizada pela rotina assistencial do hospital em questão, ou seja, $38^{\circ} \mathrm{C}$ na maioria das verificações. Com achados semelhantes, um ensaio clínico randomizado (realizado no Canadá) demonstrou que o banho de imersão, cuja temperatura da água varia de $37,8^{\circ} \mathrm{C}$ a $38,8^{\circ} \mathrm{C}$, pode reduzir as perdas de calor de neonatos a termo saudáveis em comparação ao banho de banheira. Além disso, tal cuidado auxilia os RN a manterem temperaturas corporais mais elevadas após a realização do procedimento ${ }^{(15)}$.

O primeiro banho deve ser realizado quando o RN atingir estabilidade térmica e cardiorrespiratória, sendo ideal esperar pelo menos duas horas para realizar o procedimento. Um estudo aponta que a realização do banho imediatamente após o nascimento pode comprometer a estabilidade térmica e cardiorrespiratória do recém-nascido durante o período de transição à vida extrauterina. Quando medidas de controle termal do ambiente são adotadas, a ocorrência de tais eventos é reduzida potencialmente. Com relação ao tempo de duração do banho, é recomendado que o procedimento seja tão rápido quanto for possível, sendo adequada uma duração entre cinco e dez minutos. A limitação do banho em cinco minutos previne o estresse causado pelo frio e reduz o tempo de exposição da pele dos neonatos aos agentes de limpeza ${ }^{(13,15)}$.

Foram observados 60 casos de hipotermia no estudo. Entretanto, não houve associação entre a termorregulação e as variáveis dos RN quanto ao peso e idade gestacional. Atribui-se a ausência de significância estatística entre essas, devido ao fato de ser uma amostra de neonatos saudáveis, sendo que a mediana de idade gestacional de ambos os grupos foi superior a 39 semanas e a mediana do peso foi superior a $3.300 \mathrm{~g}$, o que demostra boas condições de adaptação à vida extrauterina. Considerando a ocorrência de hipotermia nos momentos de aferição de temperatura axilar dos RN da presente pesquisa, foi observada associação com significância estatística entre a ocorrência de hipotermia e todos os momentos de verificação. Tal associação poderia ser explicada por ser um reflexo das temperaturas mais baixas das salas de parto e pelos ba- nhos terem sido realizados, em sua maioria, entre uma e duas horas de vida ${ }^{(7)}$.

O primeiro banho representa um desafio para que os RN consigam manter sua temperatura corporal adequada, porque além da exposição às variações termais do ambiente, o procedimento expõe os recém-nascidos às perdas de calor por condução e por evaporação da água. Nesse sentido, cuidados com o reaquecimento do RN após o banho são importantes. Um estudo realizado em um hospital escola dos Estados Unidos da América comprovou que o contato pele-a-pele, após o banho, pode ser uma técnica mais eficaz em seu reaquecimento em relação ao uso do berço de calor radiante, desde que a equipe preste atenção especial ao posicionamento correto do RN sobre sua mãe $e^{(13,16)}$.

Finalmente, ao realizar correlação estatística entre as variáveis termais ao que o recém-nascido foi exposto (sala de parto, sala de admissão e temperatura da água) com os quatro momentos de verificação de temperatura axilar da presente pesquisa, observou-se significância estatística (porém fraca) entre as variáveis: sala de parto e o momento quatro de verificação, ou seja, 60 minutos após o término do banho. Tal achado, também, pode ser explicado pelas baixas temperaturas admissionais do neonato na sala de parto, visto que a hipotermia neonatal é um evento que pode ocorrer tardiamente (após o banho ou horas após o nascimento) caso os cuidados termais de admissão não tenham sido eficientes ${ }^{(16)}$.

Apesar de estudos demonstrarem que a manutenção da temperatura corporal do RN pode ocorrer mesmo quando o primeiro banho é realizado com poucas horas de vida, seu adiamento proporciona diversos benefícios como o início precoce do aleitamento materno, por exemplo ${ }^{(11)}$. Nesse sentido, o adiamento de qualquer procedimento de rotina durante as primeiras horas de vida (como o primeiro banho) evita a separação do binômio mãe/RN, permite a realização do contato pele-a-pele, favorece a adaptação do mesmo ao ambiente extrauterino e promove o início da amamentação. Tais cuidados devem ser preconizados, principalmente, em localidades com poucos recursos de atendimento ao recém-nascido ${ }^{(17)}$.

\section{- CONCLUSÕES}

O presente estudo evidenciou 60 casos de hipotermia neonatal, dentre os 149 recém-nascidos participantes. Foram observados fatores protetivos do cuidado térmico prestado ao recém-nascido, tais como a existência de um cuidado de enfermagem rotineiro para a realização do procedimento, cujas orientações versam sobre o atendimento inicial do RN para os cuidados de admissão do mesmo, in- 
cluindo o controle da temperatura axilar do mesmo, controle da temperatura da água do banho e da temperatura da sala onde é realizado o procedimento.

Nosso estudo traz como contribuição, para a prática assistencial de enfermagem, evidências sobre a termorregulação do RN a termo e a prática do primeiro banho realizado com poucas horas de vida. Os resultados da pesquisa sugerem que o banho nas primeiras horas de vida dos recém-nascidos pode interferir na termorregulação, devendo ser adiado por algumas horas. Ressalta-se, também, a necessidade de maior supervisão sobre regulação da temperatura do ambiente admissional do recém-nascido, considerando-se esse um dos pilares do atendimento realizado na Golden Hour. Assim, sugere-se que maiores cuidados sobre a temperatura do RN e do ambiente sejam adotados durante o primeiro banho, devendo este ser preconizado como um cuidado protetivo com o intuito de reduzir a ocorrência de hipotermia neonatal nas primeiras horas de vida.

Recomenda-se que sejam realizados estudos comparando o banho nas primeiras horas de vida e o banho adiado por algumas horas para estabilização térmica do RN e promoção da permanência do mesmo com sua mãe.

\section{口EFERÊNCIAS}

1. Kuller JM. Update on newborn bathing. Newborn Infant Nurs Rev. 2014;14:16670. doi: https://doi.org/10.1053/j.nainr.2014.10.006.

2. Monteagudo B, Labandeira J, León-Muiñoz E, Romarís R, Ramírez-Santos A, Gozáles-vilas D, et al. Influence on neonatal and maternal factors on the prevalence of vernix caseosa. Actas Dermosifiliogr. 2011;102(9):726-9. doi: https:// doi.org/10.1016/j.ad.2011.01.006.

3. Moore E, Anderson, GC, Bergman N, Dowswell T. Early skin-to-skin contact for mother and their healthy newborn infants. Cochrane Database Syst Rev. 2012;16(5):CD003519. doi: https://doi.org/10.1002/14651858.CD003519.pub3.

4. Ministério da Saúde (BR). Secretaria de Atenção à Saúde. Portaria no 371 de 07 de maio de 2014. Institui diretrizes para a organização da atenção integral e humanizada ao recém nascido (RN) no Sistema Único de Saúde (SUS). Brasília; 2014 [citado 2017 nov 05]. Disponível em: http://bvsms.saude.gov.br/bvs/saudelegis/sas/2014/prt0371_07_05_2014.html.

5. Preer G, Pisegna JM, Cook JT, Henri AM, Phillip BL. Delaying the bath and inhospital breastfeeding rates. Breastfeed Med. 2013;8(6):480-9. doi: https://doi. org/10.1089/bfm.2012.0158.
6. Sobel HL, Silvestre MA, Mantaring JB 3rd, Oliveros YE, Nyunt-U S. Immediate newborn care practices delay thermoregulation and breastfeeding initiation. Acta Paediatr. 2011;100(8):1127-33. doi: https://doi.org/10.1111/j.16512227.2011.02215.x

7. World Health Organization (CH). Every newborn: an action plan to end preventable deaths. Geneva: WHO; 2014 [cited 2017 Oct 12]. Available from: http:// www.who.int/pmnch/about/governance/partnersforum/enap_full.pdf.

8. Ruschel, LM. Repercussões do primeiro banho na temperatura do recém-nascido [dissertação]. Porto Alegre (RS): Universidade Federal do Rio Grande do Sul; 2017.

9. American Academy of Pediatrics. Guidelines for perinatal care. 7th ed. Elk Grove Village: AAP; 2012.

10. Ministério da Saúde (BR), Conselho Nacional de Saúde. Resolução nº 466, de 12 de dezembro de 2012. Diretrizes e normas regulamentadoras de pesquisas envolvendo seres humanos. Diário Oficial da União [da] República Federativa do Brasil. 2013 jun 13;150(112 Seção 1):59-62.

11. Lund $\mathrm{CH}$. Bathing and beyond: current bathing controversies for newborn infants. Adv Neonatal Care. 2016;16(5S):S13-S20. doi: https://doi.org/10.1097/ ANC.0000000000000336.

12. Salasibew MM. et al. Measurement of delayed bathing and early initiation of breastfeeding: a cross-sectional survey exploring experiences of data collectors in Ethiopia. BMC Pediatr. 2015;15:35. doi: https://doi.org/10.1186/s12887015-0350-7.

13. Lund C, Brandon D, Holden A, Kuller J, Hill C, Bingham D, et al. Evidence-based clinical practice guideline: neonatal skin care. 3rd ed. Washington, DC: Association of Women's Health, Obstetric and Neonatal Nurses; 2013.

14. Duryea EL, Nelson DB, Wyckoff MH, Grant EN,Tao W, Sadana N, et al. The impact of ambient operating room temperature on neonatal and maternal hypothermia and associated morbidities: a randomized controlled trial. Am J Obstet Gynecol. 2016;214(4):505.e1-505.e7. doi: https://doi.org/10.1016/j. ajog.2016.01.190.

15. Albuquerque RS, Mariani Neto C, Bersusa AAS, Dias VM, Silva MIM. Temperatura dos recém-nascidos submetidos ao calor radiante e ao dispositivo Top Maternal ao nascimento. Rev Latino-Am Enfermagem. 2016;24:e2741. doi: https://doi. org/10.1590/1518-8345.0305.2741.

16. George S, Phillips K, Mallory S, Holmquistova I, Hare R, Allen S, et al. A pragmatic descriptive study of rewarming the newborn after the first bath. J Obstet Gynecol Neonatal Nurs. 2015;44(2):203-9. doi: https://doi.org/10.1111/15526909.12556.

17. Blume-Peytavi U, Lavender T, Jenerowicz D, Ryumina I, Stalder JF, Torrelo A, et al. Recommendations from a European roundtable meeting on best practice health infant skin-care. Pediatr Dermatol. 2016;33(3):311-21. doi: https://doi. org/10.1111/pde.12819.

Recebido: 20.12.2017

Aprovado: 02.07.2018 